\title{
Prevention and Therapy of Type 2 Diabetes-What Is the Potential of Daily Water Intake and Its Mineral Nutrients?
}

\author{
Johannes Naumann *, Diana Biehler, Tania Lüty and Catharina Sadaghiani \\ Interdisciplinary Center for Treatment and Research in Balneology, Institute for Infection Prevention and \\ Hospital Epidemiology, Medical Center-University of Freiburg, Faculty of Medicine, University of Freiburg, \\ 79106 Freiburg, Germany; diana.biehler@uniklinik-freiburg.de (D.B.); tania.luety@uniklinik-freiburg.de (T.L.); \\ catharina.sadaghiani@uniklinik-freiburg.de (C.S.) \\ * Correspondence: johannes.naumann@uniklinik-freiburg.de; Tel.: +49-761-270-82430
}

Received: 26 June 2017; Accepted: 18 August 2017; Published: 22 August 2017

\begin{abstract}
We aim to present an overview of the possible influence of drinking water in general and mineral water in particular in improving glycemic parameters in persons with or without type 2 diabetes. We performed a literature search that produced 15 randomized controlled trials (RCTs) on this topic with mainly small sample sizes. We also discuss relevant observational and animal studies as well as the effects of important supplements in mineral water such as hydrogencarbonate and magnesium. There is low evidence for the positive effects of water or mineral water in improving glycemic parameters in diabetic and non-diabetic persons, and the results are heterogenous, making it difficult to reach an unequivocal conclusion. Meta-analyses of prospective cohort studies and other observational studies, studies with animal models and interventional studies using hydrogencarbonate and magnesium supplements suggest a probable positive effect of drinking water and mineral water in particular on glycemic parameters, supporting the positive results found in some of the RCTs, especially those substituting diet beverages or caloric beverages with water, or those using bicarbonate and magnesium-rich water. Regarding the high prevalence, the associated suffering and the resulting health expenditures of type 2 diabetes, it is imperative to conduct larger and more rigorous trials to answer the question whether drinking water or mineral water can improve glycemic parameters in diabetic and non-diabetic persons.
\end{abstract}

Keywords: diabetes; water intake; mineral water; magnesium; bicarbonate; review; prevention

\section{Introduction}

The incidence of type 2 diabetes mellitus, pre-diabetes, impaired glucose tolerance and metabolic syndrome has increased considerably worldwide. The global prevalence of diabetes is estimated to be $8.5 \%$, i.e., there are 422 million diabetics worldwide and 59.8 million diabetics in Europe $(9.1 \%)$ alone $[1,2]$.

Making lifestyle changes including a healthy diet and sufficient physical activity of $150 \mathrm{~min}$ a week, which should result in a weight loss of at least 7\% [3], is the most effective way to reduce the risk of developing diabetes by an estimated $34-43 \%$. Furthermore, a diet and physical activity can reduce diabetes incidence, cardiovascular (hazard ratio (HR) 0.59, 95\% confidence interval (CI) 0.36-0.96; $p=0.033$ ) and all-cause mortality (HR 0.71, 95\% CI 0.51-0.99; $p=0.049$ ) [4].

A healthy diet should include beverages with low sugar and calorie content, but not diet (artificially sweetened) beverages, because habitual consumption of sugar sweetened drinks, sweetened-milk beverages [5] and diet beverages [6] is associated with higher type 2 diabetes risk. 
People who don't drink sugar sweetened beverages tend to have higher quality diets and do not compensate for sugar or energy deficits by consuming more sugary foods [7].

A nutrition survey examining the relationship between the quantity of water taken in and $\mathrm{HbA} 1 \mathrm{c}$ in 1035 participants calculated that an increase of $240 \mathrm{~mL}$ in daily water intake reduces the risk of elevated $\mathrm{HbAc} 1>5.5 \%$ by $22 \%$ in men but not in women [8].

A review of 134 randomized controlled trials (RCTs), where water consumption was increased right before or during a meal, showed heterogenic effects on energy intake, energy expenditure, fat oxidation and weight change [9].

Moreover, mineral nutrients such as bicarbonate [10] and magnesium [11], in particular, are thought to affect glucose metabolism. An increased dietary acid load is associated with the development of insulin resistance [12], while a pre-existing diabetes favors acidosis, which, in turn, leads to increased insulin resistance [13].

Drinking recommendations are less common and detailed than food recommendations [3,14], and there is little scientific evidence, and, to our knowledge, no review of RCTs on drinking water and its influence on glycemic parameters, respectively type 2 diabetes.

This review aims to explore to what extent drinking water with low or high mineralization for at least four weeks influences glycemic parameters in healthy or type 2 diabetes participants in daily life situations as compared with other drinks. The results of the RCTs found in the literature search will be discussed alongside observational studies, animal studies, pathophysiological aspects as well as results from interventional studies using mineral supplements.

\section{Methods}

\subsection{Overview}

Two reviewers (J.N., D.B.) identified RCTs as well as crossover RCTs reporting long-term ( $\geq 4$ weeks treatment) effects of drinking water on glycemic parameters.

\subsection{Study Search}

The Medline database was searched via PubMed for fully published peer-reviewed articles from the time of database inception to 8 August 2017. The search strategy is outlined in Table 1. A systematic literature search was not performed since the focus was to evaluate the potential, not to estimate unbiased effects of drinking water and its mineral nutrients in prevention and therapy of type 2 diabetes.

Table 1. Search strategy.

\begin{tabular}{|c|c|c|c|}
\hline Search No. & Search Terms & Results & Search Fields \\
\hline 1 & water & 819,017 & All Fields \\
\hline 2 & glucose or glycemic or glycaemic or cholesterol & 733,923 & All Fields \\
\hline 3 & drink $^{*}$ or consumption or beverage ${ }^{*}$ & 188,224 & All Fields \\
\hline 4 & study or trial ${ }^{*}$ or review & $10,225,821$ & All Fields \\
\hline 5 & $\begin{array}{c}\text { humans or women or men } \\
((((\text { water) AND ((glucose OR glycemic OR glycaemic }\end{array}$ & $16,761,909$ & All Fields \\
\hline 6 & $\begin{array}{l}\text { OR cholesterol))) AND ((drink* OR consumption OR } \\
\text { beverage*))) AND ((study OR trial* OR review))) AND } \\
\text { (humans OR women OR men) }\end{array}$ & 1139 & All Fields \\
\hline
\end{tabular}

\subsection{Study Inclusion and Exclusion Criteria}

The criteria were as follows:

(1) Types of study: RCTs were the focus of this review to restrict potential sources of error and facilitate inference about possible causal mechanisms. They were included regardless of quality measures, such as double-blind design, complete protocol adherence, patient attrition, similarity 
of treatment and control groups at baseline and intention-to-treat analysis or sample size, target population or unit of analysis (e.g., individual or group-level data). They were only eligible if they were published as full paper articles. No language restrictions were made.

(2) Types of participants: individuals of all ages (whether healthy or with or without type 2 diabetes, at risk of type 2 diabetes or other diseases, as having cardiovascular risk factors (CVRF), being overweight or obese or not, with hyperlipemia or not; excluded: type 1 diabetes).

(3) Types of intervention: plain or drinking water, served at any temperature, whether tap or bottled water, high or low mineralized, carbonated or uncarbonated water.

(4) Types of comparisons: interventions or control groups with drinking water tested alone, as a single intervention or as main part of a multi-component intervention for at least four weeks.

(5) Types of outcome: at least one parameter for glycemic control as shown in Table 2 independent whether it was a primary or secondary outcome of the study.

Studies excluded were those that measured only short-term effects of drinking water on glycemic parameters because short-term effects may not well reflect the influence on incidence and outcome of type 2 diabetes. Studies investigating severe diseases or special situations, such as, for example, acute gastroenteritis or extreme sport or climatic situations were also excluded.

Table 2. Parameters for glycemic control.

Parameters (with Normal Values) for Glycemic Control Reported in the Studies
Fasting plasma glucose $(\mathrm{FPG}<110 \mathrm{mg} / \mathrm{dL})$
Flucose tolerance test $($ level $2 \mathrm{~h}$ after glucose loading $<140 \mathrm{mg} / \mathrm{dL})$
Fasting insulin $(6-25 \mathrm{mU} / \mathrm{L})$
HOMA-IR $(<2)($ Homeostatic Model Assessment for Insulin Resistance)
(Product of fasting insulin $(\mu \mathrm{U} / \mathrm{mL}) \times$ fasting glucose $(\mathrm{mg} / \mathrm{dL}) / 405)$
Glycated hemoglobin, HbA1c $(<\mathbf{6} \%)$
(Measure for blood glucose levels during the last 1 to 3 months)
Glycoalbumin $(\mathbf{1 1 - 1 6 \% )}$
(Measure for blood glucose levels in the last 2 to 4 weeks)
Fructosamin $(\mathbf{2 0 0 - 2 9 0} \mu$ mol/L)
(Measure for blood glucose levels in the last 1 to 3 weeks)

\subsection{Data Extraction and Risk of Bias Evaluation}

The authors (J.N., D.B.) of the review present here independently extracted relevant study information (for example, population, characteristics of the intervention and control, outcome measures, results) using predefined data sheets, including the Cochrane risk-of-bias indicators (J.N., C.S.) [15]. We report the items which are of high or unclear risk of bias regarding the key domains-selection, performance, detection, attrition and reporting bias. If necessary, existing inconsistencies were solved by discussion, and consensus achieved.

\section{Results}

\subsection{Literature Search}

Our literature search yielded 1139 articles, 1078 of which were excluded by two independent reviewers (J.N., D.B.) based on their titles (other intervention or outcomes or study type), thus leaving 61 trials (Figure 1).

After reading the abstracts, and, if necessary, the whole article, another 47 trials were excluded:

(a) Population: 1 (type 1 diabetes)

(b) Comparison: 41 trials only investigated short-term effects ( $<4$-weeks treatment)

(c) Outcome: 1 (glycemic parameter measured, but not reported) 
(d) Publication: 3 only abstracts (1 Russian, 1 Japanese, 1 Italian)

(e) Double publishing: 1

After reviewing references, one additional RCT [16] was included, which is not indexed in Medline or web of science. Hence, 15 studies were reviewed.

\subsection{Description of Included Studies}

The characteristics of the included studies are detailed in the following Tables 3-5.

The studies were separated according to mineralization of the water and the control group:

Table 3: RCTs comparing water with other drinks or no drink (eight studies); [17-24].

Table 4: RCTs comparing low mineralized with bicarbonate-rich water (five studies); [25-29].

Table 5: RCTs comparing low mineralized with bicarbonate- and magnesium-rich water (two studies); [16,30].

Regarding the study populations investigated, we included four studies with type 2 diabetes $[19,22-24]$, three with overweight or obese $[17,18,21]$, three with moderately elevated cholesterol $[26,27,29]$, one with at least one CVRF [20] and four with healthy participants $[16,25,28,30]$.

Three studies investigated women $[18,19,25]$, one of them postmenopausal women [25], two studies men [16,29] and ten studies both genders [17,20-24,26-28,30]. One study investigated elderly people (60-72 years) [28].

Of the included studies, eight compared water with other beverages (Table 3): diet beverages [17-19], artificially sweetened cola, sucrose sweetened cola and semiskim milk [21], blueberry juice or no water [20], red or white wine [23], red wine with or without alcohol [22], and chamomile tea [24].

A further seven studies compared different types of water, all low mineralized with bicarbonate-rich water $(879-2946 \mathrm{mg} / \mathrm{L})$, five of them with low [25-29] and two [16,17,30] with high concentrations of magnesium (102 and $291 \mathrm{mg} / \mathrm{L}$ ) (Tables 4 and 5); three studies compared uncarbonated water $[16,28,30]$, two studies carbonated water $[27,29]$ and two studies carbonated bicarbonate-rich water versus uncarbonated mineral water $[25,26]$.

All 15 studies reported fasting blood glucose, 12 studies fasting insulin or C-peptide levels (not reported in $[17,25,29]$ ) of which seven calculated HOMA-IR; (Homeostatic Model Assessment for Insulin Resistance) $[18,19,21-24,30]$. Four of the studies additionally conducted glucose tolerance tests (GTT) $[16,18,19,28]$, one reported serum fructosamin concentrations [16], and one plasma glycoalbumin concentration [30].

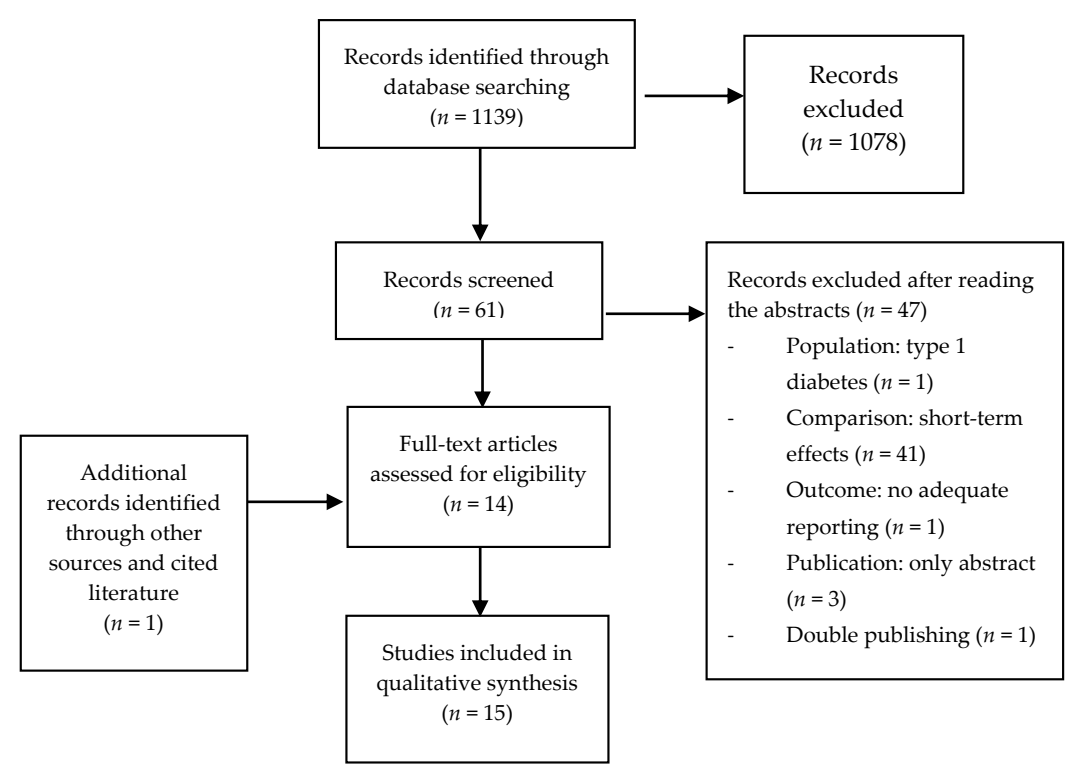

Figure 1. Flow-chart of the literature search. 
Table 3. Randomized controlled trials (RCTs) comparing water with other drinks or no drink.

\begin{tabular}{|c|c|c|c|c|c|c|c|}
\hline Author/y & Study Type & Population & Intervention & Comparison & Outcome & Results & Risk of Bias \\
\hline $\begin{array}{l}\text { Tate et al., } \\
2012 \text { [17] }\end{array}$ & $\begin{array}{l}\text { RCT } \\
\text { parallel } \\
3 \text { groups }\end{array}$ & $\begin{array}{l}318 \text { obese, BMI } 36.3 \mathrm{~kg} / \mathrm{m}^{2}, \\
84 \% \text { female, medium age } \\
42 \mathrm{y} \text {, USA }\end{array}$ & $\begin{array}{l}\text { Advice to replace more } \\
\text { than } 2 \text { servings (each } \\
350-500 \mathrm{~mL} \text { ) of caloric } \\
\text { beverages per day with } \\
\text { water for six months }\end{array}$ & $\begin{array}{l}\text { CG1: Advice to } \\
\text { replace more than } 2 \\
\text { servings of caloric } \\
\text { beverages per day } \\
\text { with diet beverages } \\
\text { CG2: no advice to } \\
\text { change beverages, but } \\
\text { general nutritional } \\
\text { weight loss advices }\end{array}$ & $\begin{array}{l}\text { FPG (SO) } \\
\text { Weight loss (PO) }\end{array}$ & $\begin{array}{l}\text { Water group had a sign. Increase of } \\
\text { water consumption of } 1 \mathrm{~L} \text { after } 3 \\
\text { months and } 0.8 \mathrm{~L} \text { after } 6 \text { months } \\
\text { and a sign. Decrease of FPG } \\
\text { compared to control group with no } \\
\text { change of beverages after } 3 \text { and } 6 \\
\text { months. }\end{array}$ & $\begin{array}{l}\text { ITT, drop-outs } \\
33 / 318 \text { after } 3 \\
\text { months and } 46 / 318 \\
\text { after } 6 \text { months, no } \\
\text { blinding }\end{array}$ \\
\hline $\begin{array}{l}\text { Madjd et al., } \\
2015 \text { [18] }\end{array}$ & $\begin{array}{l}\text { RCT } \\
\text { parallel } \\
2 \text { groups } \\
\text { single-blind }\end{array}$ & $\begin{array}{l}62 \text { healthy overweight and } \\
\text { obese women, BMI } 27-40 \\
\mathrm{~kg} / \mathrm{m}^{2}, 18-50 \mathrm{y} \text {, } \\
\text { non-smokers, participating } \\
\text { in a weight loss program, } \\
\text { Iran }\end{array}$ & $\begin{array}{l}250 \mathrm{~mL} \text { tap water (not } \\
\text { specified) per day after } \\
\text { lunch for } 24 \text { weeks }\end{array}$ & $250 \mathrm{~mL}$ diet beverage & $\begin{array}{l}\text { FPG } \\
\text { Fasting insulin } \\
\text { HOMA-IR } \\
\text { GTT } \\
\text { Body weight } \\
\text { (all PO) }\end{array}$ & $\begin{array}{l}\text { Water group sign. more weight loss } \\
(8.8 \mathrm{~kg} \text { vs. } 7.6 \mathrm{~kg} p=0.015) \\
\text { lower FPG }(2.8 \mathrm{mU} / \mathrm{L} \text { vs. } 1.7 \mathrm{mU} / \mathrm{L} \\
p<0.001) \text { better insulin sensitivity } \\
\text { (HOMA-IR } 1 \text { vs. } 0.8 p<0.001) \\
\text { better GTT }(1 \mathrm{mmol} / \mathrm{L} \text { vs. } 0.7 \\
\text { mmol/L, } p<0.001) \text { compared to } \\
\text { control group after } 24 \text { weeks. }\end{array}$ & $\begin{array}{l}\text { No ITT, } 9 / 71 \\
\text { drop-outs, measures } \\
\text { of blinding not } \\
\text { reported }\end{array}$ \\
\hline $\begin{array}{l}\text { Madjd et al., } \\
2017 \text { [19] }\end{array}$ & $\begin{array}{l}\text { RCT } \\
\text { parallel } \\
2 \text { groups } \\
\text { single-blind }\end{array}$ & $\begin{array}{l}81 \text { overweight and obese } \\
\text { women, BMI } 27-35 \mathrm{~kg} / \mathrm{m}^{2} \\
\text { with type } 2 \text { diabetes HbA1c } \\
6.5-7.2 \% \text {, age } 18-50 \mathrm{y}, \\
\text { non-smokers, participating } \\
\text { in a weight loss program } \\
\text { with only metformin, Iran }\end{array}$ & $\begin{array}{l}250 \mathrm{~mL} \text { tap water (not } \\
\text { specified) per day after } \\
\text { lunch for } 24 \text { weeks }\end{array}$ & $250 \mathrm{~mL}$ diet beverage & $\begin{array}{l}\text { FPG } \\
\text { Fasting insulin } \\
\text { HOMA-IR } \\
\text { GTT } \\
\text { Body weight } \\
\text { (all PO) }\end{array}$ & $\begin{array}{l}\text { Water group sign. more weight loss } \\
(-6.40 \text { vs. }-5.25 \mathrm{~kg} p=0.006) ; \\
\text { lower FPG }(-1.63 \text { vs. }-1.29 \\
\text { mmol/L } p=0.06) \\
\text { lower fasting insulin }(-5.71 \text { vs. } \\
-4.16 \mathrm{mU} / \mathrm{L} p<0.011) \\
\text { better insulin sensitivity } \\
\text { (HOMA-IR }-3.2 \text { vs. }-2.48 p< \\
0.003) \\
\text { better GTT ( }-1.67 \text { vs. }-1.35 \\
\text { mmol/L } p=0.027) \\
\text { Compared to the control group } \\
\text { after } 24 \text { weeks. }\end{array}$ & $\begin{array}{l}\text { ITT, } 16 / 81 \\
\text { drop-outs, measures } \\
\text { of blinding not } \\
\text { reported }\end{array}$ \\
\hline $\begin{array}{l}\text { Tonstad et al., } \\
2006 \text { [20] }\end{array}$ & $\begin{array}{l}\text { RCT } \\
\text { parallel } \\
3 \text { groups }\end{array}$ & $\begin{array}{l}67 \text { men and } 27 \\
\text { post-menopausal women } \\
\text { (total } n=94 \text { ) } \\
\text { with at least } 1 \text { CVRF, } \\
\text { Norway }\end{array}$ & $\begin{array}{l}1 \mathrm{~L} \text { tap water (not } \\
\text { specified) per day for } 4 \\
\text { weeks }\end{array}$ & $\begin{array}{l}\text { CG1: } 1 \text { L blueberry } \\
\text { juice } \\
\text { CG2: no change of } \\
\text { habits }\end{array}$ & $\begin{array}{l}\text { FPG } \\
\text { Fasting insulin } \\
\text { C-peptide } \\
\text { (all SO) } \\
\text { Blood viscosity } \\
\text { (PO) }\end{array}$ & $\begin{array}{l}\text { No sign. difference between groups } \\
\text { after } 4 \text { weeks (exact data not } \\
\text { presented) }\end{array}$ & $\begin{array}{l}\text { No ITT, } 5 / 99 \\
\text { drop-outs, no } \\
\text { blinding, }\end{array}$ \\
\hline
\end{tabular}


Table 3. Cont.

\begin{tabular}{|c|c|c|c|c|c|c|c|}
\hline Author/y & Study Type & Population & Intervention & Comparison & Outcome & Results & Risk of Bias \\
\hline $\begin{array}{l}\text { Maersk et al., } \\
2012 \text { [21] }\end{array}$ & $\begin{array}{l}\mathrm{RCT} \\
\text { parallel } \\
2 \text { groups }\end{array}$ & $\begin{array}{l}47 \text { healthy overweight } \\
\text { adults, } 20-50 \text { y, BMI } 26-40 \\
\mathrm{~kg} / \mathrm{m}^{2}, \mathrm{RR}<160 / 100 \\
\text { Denmark }\end{array}$ & $\begin{array}{l}1 \mathrm{~L} \text { uncarbonated water } \\
\text { per day for } 6 \text { months } \\
\text { Aqua d'or } \\
\mathrm{HCO}^{3-} 71 \mathrm{mg} / \mathrm{L} \\
\mathrm{Cl}^{-} 16.5 \mathrm{mg} / \mathrm{L} \\
\mathrm{Na}^{+} 14.9 \mathrm{mg} / \mathrm{L} \\
\mathrm{Mg}^{2+} 6 \mathrm{mg} / \mathrm{L}\end{array}$ & $\begin{array}{l}1 \mathrm{~L} \text { regular Cola } \\
\text { (sucrose-sweetened) } \\
1 \mathrm{~L} \text { diet Cola, } \\
\text { aspartame-sweetened } \\
1 \mathrm{~L} \text { semi-skim milk }\end{array}$ & $\begin{array}{l}\text { FPG } \\
\text { Fasting insulin } \\
\text { HOMA-IR } \\
\text { (all SO) }\end{array}$ & $\begin{array}{l}\text { No sign. difference for FPG } \\
\text { Insulin and HOMA-IR between } \\
\text { groups }\end{array}$ & $\begin{array}{l}\text { No ITT, 13/60 } \\
\text { drop-outs, no } \\
\text { blinding, sign. } \\
\text { difference for sex at } \\
\text { baseline (adjusted in } \\
\text { analysis), } \\
\text { randomization and } \\
\text { allocation not } \\
\text { reported }\end{array}$ \\
\hline $\begin{array}{l}\text { Mori et al., } \\
2016 \text { [22] }\end{array}$ & $\begin{array}{l}\text { RCT } \\
\text { crossover } \\
3 \text { groups }\end{array}$ & $\begin{array}{l}24 \text { well controlled type } 2 \\
\text { diabetes HbA1c }<8.5 \%, 19 \\
\text { men, } 5 \text { women, } 40-70 \mathrm{y}, \\
\text { regular alcohol intake } \\
\text { women } 20-30 \mathrm{~g} / \text { day, men } \\
30-40 \mathrm{~g} / \text { day, Australia }\end{array}$ & $\begin{array}{l}\text { Tap water (not specified) } \\
\text { women } 230 \mathrm{~mL} \text { per day } \\
\text { men } 300 \mathrm{~mL} \text { per day for } \\
4 \text { weeks }\end{array}$ & $\begin{array}{l}\text { CG1: red wine } \\
\text { women } 230 \mathrm{~mL} / \text { day } \\
\text { ( } 24 \mathrm{~g} \text { alcohol/day) } \\
\text { Men } 300 \mathrm{~mL} / \text { day ( } 31 \\
\text { g alcohol/day), } \\
\text { CG2: dealcoholized } \\
\text { red wine } \\
\text { women } 230 \mathrm{~mL} / \text { day } \\
\text { Men } 300 \mathrm{~mL} / \text { day }\end{array}$ & $\begin{array}{l}\text { FPG } \\
\text { Fasting Insulin } \\
\text { HOMA-IR } \\
\text { (all SO) } \\
\text { CVRF (PO) }\end{array}$ & $\begin{array}{l}\text { No sign. difference for FPG } \\
\text { Insulin and HOMA-IR between } \\
\text { groups }\end{array}$ & $\begin{array}{l}\text { No ITT, } 4 / 28 \\
\text { drop-outs, no } \\
\text { blinding }\end{array}$ \\
\hline $\begin{array}{l}\text { Gepner et al., } \\
2015 \text { [23] }\end{array}$ & $\begin{array}{l}\text { RCT parallel } 3 \\
\text { groups }\end{array}$ & $\begin{array}{l}224 \text { alcohol-abstaining } \\
\text { adults type } 2 \text { diabetes, } \\
\text { HbA1c } 6.4-10 \% \text {, Israel }\end{array}$ & $\begin{array}{l}150 \mathrm{~mL} \text { mineral water) } \\
\text { per day in the evening } \\
\text { for } 2 \text { years } \\
\text { Mey Eden, different } \\
\text { European sources, all } \\
\text { low mineralized }\end{array}$ & $\begin{array}{l}\text { CG1: } 150 \mathrm{~mL} \text { red wine } \\
\text { CG2: } 150 \mathrm{~mL} \text { white } \\
\text { wine }\end{array}$ & $\begin{array}{l}\text { FPG } \\
\text { HOMA-IR (all PO) } \\
\text { Fasting insulin } \\
\text { HbA1c (all SO) } \\
\text { HDL-Chol, } \\
\text { Apolip-a (PO) }\end{array}$ & $\begin{array}{l}\text { Water group sign. Increase of FPG } \\
\text { and HOMA-IR compared to white } \\
\text { wine. No sign. difference for } \\
\text { HbA1c, fasting insulin and } \\
\text { outcomes compared to red wine }\end{array}$ & ITT, no blinding \\
\hline $\begin{array}{l}\text { Rafraf et al., } \\
2015 \text { [24] \& } \\
\text { Zemestani et } \\
\text { al., 2016 [31] }\end{array}$ & $\begin{array}{l}\text { RCT } \\
\text { parallel } \\
2 \text { groups } \\
\text { single-blind }\end{array}$ & $\begin{array}{l}64 \text { type } 2 \text { diabetes (males } \\
\text { and females) } \\
\text { aged } 30-60 \mathrm{y} \\
\text { Iran }\end{array}$ & $\begin{array}{l}150 \mathrm{~mL} \text { hot water three } \\
\text { times per day } \\
\text { immediately after meals } \\
\text { for } 8 \text { weeks. }\end{array}$ & $\begin{array}{l}150 \mathrm{~mL} \text { chamomile tea } \\
(3 \mathrm{~g} / 150 \mathrm{~mL} \text { hot } \\
\text { water })\end{array}$ & $\begin{array}{l}\text { FPG } \\
\text { Fasting Insulin } \\
\text { HOMA-IR } \\
\text { HbA1c }\end{array}$ & $\begin{array}{l}\text { Chamomile tea group sign. } \\
\text { Decrease for HbA1c }(p=0.023), \\
\text { serum insulin levels }(p<0.001) \\
\text { HOMA-IR }(p<0.001) \text {, and no sign. } \\
\text { difference for FPG compared to hot } \\
\text { water }\end{array}$ & $\begin{array}{l}\text { ITT, no drop-outs, } \\
\text { single-blind, sign. } \\
\text { difference for FPG, } \\
\text { insulin and } \\
\text { HOMA-IR at } \\
\text { baseline, no PO } \\
\text { specified }\end{array}$ \\
\hline
\end{tabular}

CG: control group; PO: primary ou 
Table 4. Randomized controlled trials (RCTs) comparing low mineralized water with bicarbonate-rich water with low magnesium.

\begin{tabular}{|c|c|c|c|c|c|c|c|}
\hline Author/y & Study Type & Population & Intervention & Comparison & Outcome & Results & Risk of Bias \\
\hline $\begin{array}{l}\text { Schoppen et } \\
\text { al., } 2004 \text { [25] }\end{array}$ & $\begin{array}{l}\text { RCT crossover } \\
2 \text { groups }\end{array}$ & $\begin{array}{l}18 \text { healthy women, } \\
>1 \text { postmenopausal, } \\
\text { BMI }<30 \mathrm{~kg} / \mathrm{m}^{2} \\
\text { Spain }\end{array}$ & $\begin{array}{l}1 \mathrm{~L} \text { per day for } 2 \text { months } \\
\text { Bicarbonate-rich carbonated water: } \\
\mathrm{HCO}^{3-} 2094 \mathrm{mg} / \mathrm{L} \\
\mathrm{Cl}^{-} 583 \mathrm{mg} / \mathrm{L} \\
\mathrm{Na}^{+} 1116 \mathrm{mg} / \mathrm{L} \\
\mathrm{Mg}^{2+} 6 \mathrm{mg} / \mathrm{L}\end{array}$ & $\begin{array}{l}\text { Uncarbonated } \\
\text { water: } \\
\mathrm{HCO}^{3-} 71 \mathrm{mg} / \mathrm{L} \\
\mathrm{Cl}^{-} 6 \mathrm{mg} / \mathrm{L} \\
\mathrm{Na}^{+} 9 \mathrm{mg} / \mathrm{L} \\
\mathrm{Mg}^{2+} 3 \mathrm{mg} / \mathrm{L}\end{array}$ & $\begin{array}{l}\text { FPG (SO) } \\
\text { CVRF (PO) }\end{array}$ & $\begin{array}{l}\text { Bicarbonate rich water sign. }(p< \\
0.001) \text { decreased FPG compared to } \\
\text { control water }(5.54 \mathrm{vs.} 5.17 \mathrm{mmol} / \mathrm{L})\end{array}$ & $\begin{array}{l}\text { ITT, } 0 / 18 \\
\text { drop-outs, no } \\
\text { blinding, } \\
\text { randomization } \\
\text { and allocation not } \\
\text { reported }\end{array}$ \\
\hline $\begin{array}{l}\text { Toxqui \& } \\
\text { Vaquero, } 2016 \\
\text { [26] }\end{array}$ & $\begin{array}{l}\text { RCT crossover } \\
2 \text { groups } \\
\text { single-blind }\end{array}$ & $\begin{array}{l}64 \text { healthy adults } \\
18-45 \mathrm{y} \text {, with } \\
\text { moderately elevated } \\
\text { cholesterol (5.2-7.8 } \\
\text { mmol/L), Spain }\end{array}$ & $\begin{array}{l}1 \mathrm{~L} \text { of the test water per day for } 8 \\
\text { weeks followed by an } 8 \text {-week } \\
\text { washout period } \\
\text { Bicarbonate-rich carbonated water: } \\
\mathrm{HCO}^{3-} 2050 \mathrm{mg} / \mathrm{L} \\
\mathrm{Cl}^{-} 622 \mathrm{mg} / \mathrm{L} \\
\mathrm{Na}^{+} 1090 \mathrm{mg} / \mathrm{L} \\
\mathrm{Mg}^{2+} 5.8 \mathrm{mg} / \mathrm{L}\end{array}$ & $\begin{array}{l}\text { Uncarbonated } \\
\text { water: } \\
\mathrm{HCO}^{3-} 75 \mathrm{mg} / \mathrm{L} \\
\mathrm{Cl}^{-} 4.8 \mathrm{mg} / \mathrm{L} \\
\mathrm{Na}^{+} 7.6 \mathrm{mg} / \mathrm{L} \\
\mathrm{Mg}^{2+} 2.8 \mathrm{mg} / \mathrm{L}\end{array}$ & $\begin{array}{l}\text { FPG } \\
\text { Fasting insulin } \\
\text { (all SO) } \\
\text { Cholesterol (PO) }\end{array}$ & $\begin{array}{l}\text { No sign. difference for FPG } \\
\text { and fasting insulin between groups } \\
\text { Sign. reduction of fasting glucose } \\
\text { and LDL-cholesterol compared to } \\
\text { baseline in both groups. }\end{array}$ & $\begin{array}{l}\text { No ITT, } 8 / 72 \\
\text { drop-outs, no } \\
\text { blinding, }\end{array}$ \\
\hline $\begin{array}{l}\text { Pérez-Granados } \\
\text { et al., } 2010 \text { [27] }\end{array}$ & $\begin{array}{l}\text { RCT crossover } \\
2 \text { groups } \\
\text { single-blind }\end{array}$ & $\begin{array}{l}18 \text { healthy adults } \\
18-40 \mathrm{y} \text {, with } \\
\text { moderately elevated } \\
\text { cholesterol }(>5.2 \\
\text { mmol/L), Spain }\end{array}$ & $\begin{array}{l}1 \mathrm{~L} \text { of the test water per day for } 8 \\
\text { weeks followed by an } 8 \text {-week } \\
\text { washout period } \\
\text { Bicarbonate-rich carbonated water: } \\
\mathrm{HCO}^{3-} 2120 \mathrm{mg} / \mathrm{L} \\
\mathrm{Cl}^{-} 597 \mathrm{mg} / \mathrm{L} \\
\mathrm{Na}^{+} 1102 \mathrm{mg} / \mathrm{L} \\
\mathrm{Mg}^{2+} 9.4 \mathrm{mg} / \mathrm{L}\end{array}$ & $\begin{array}{l}\text { Carbonated water: } \\
\mathrm{HCO}^{3-} 104 \mathrm{mg} / \mathrm{L} \\
\mathrm{Cl}^{-} 11 \mathrm{mg} / \mathrm{L} \\
\mathrm{Na}^{+} 8.7 \mathrm{mg} / \mathrm{L} \\
\mathrm{Mg}^{2+} 5.0 \mathrm{mg} / \mathrm{L}\end{array}$ & $\begin{array}{l}\text { FPG } \\
\text { Fasting insulin } \\
\text { (all SO) } \\
\text { Cholesterol (PO) }\end{array}$ & $\begin{array}{l}\text { Bicarbonate-rich water decreased } \\
\text { FPG not sign. }(p=0.056) \text { and no sign. } \\
\text { difference for fasting insulin } \\
\text { compared to control water. }\end{array}$ & $\begin{array}{l}\text { No ITT, } 10 / 28 \\
\text { drop-outs, single } \\
\text { blind, } \\
\text { randomization } \\
\text { and allocation not } \\
\text { reported }\end{array}$ \\
\hline $\begin{array}{l}\text { Schorr et al., } \\
1996 \text { [28] }\end{array}$ & $\begin{array}{l}\text { RCT crossover } \\
3 \text { groups } \\
\text { double-blind }\end{array}$ & $\begin{array}{l}16 \text { healthy adults } \\
60-72 \mathrm{y} \text {, salt reduced } \\
\text { diet }(<2.3 \mathrm{~g} / \text { day }) \\
\text { Germany }\end{array}$ & $\begin{array}{l}1.5 \mathrm{~L} \text { of the test water per day for } 4 \\
\text { weeks with a 2-week washout } \\
\text { period before the control water } \\
\text { Bicarbonate-rich water: } \\
\text { (1.) } \mathrm{HCO}^{3-} 879 \mathrm{mg} / \mathrm{L} \\
\mathrm{Cl}^{-} 1507 \mathrm{mg} / \mathrm{L} \\
\mathrm{Na}+1295 \mathrm{mg} / \mathrm{L} \\
\mathrm{Mg}^{2+} 9.7 \mathrm{mg} / \mathrm{L} \\
(2 .) \mathrm{HCO}^{3-} 1983 \mathrm{mg} / \mathrm{L} \\
\mathrm{Cl}^{-} 152 \mathrm{mg} / \mathrm{L} \\
\mathrm{Na}^{+} 602 \mathrm{mg} / \mathrm{L} \\
\mathrm{Mg}^{2+} 53 \mathrm{mg} / \mathrm{L}\end{array}$ & $\begin{array}{l}\text { Uncarbonated } \\
\text { water: } \\
\mathrm{HCO}^{3-}<5 \mathrm{mg} / \mathrm{L} \\
\mathrm{Cl}^{-}<5 \mathrm{mg} / \mathrm{L} \\
\mathrm{Na}^{+}<5 \mathrm{mg} / \mathrm{L} \\
\mathrm{Mg}^{2+}<1 \mathrm{mg} / \mathrm{L}\end{array}$ & $\begin{array}{l}\text { FPG } \\
\text { Fasting insulin } \\
\text { GTT } \\
\text { (all PO) }\end{array}$ & $\begin{array}{l}\text { No sign. difference between the } \\
\text { groups. }\end{array}$ & $\begin{array}{l}\text { No ITT, } 5 / 21 \\
\text { drop-outs }\end{array}$ \\
\hline $\begin{array}{l}\text { Zair et al., } 2013 \\
\text { [29] }\end{array}$ & $\begin{array}{l}\text { RCT crossover } \\
2 \text { groups } \\
\text { double-blind }\end{array}$ & $\begin{array}{l}12 \text { healthy men, } \\
20-60 \mathrm{y}, \mathrm{BMI} 18.5-25 \\
\mathrm{~kg} / \mathrm{m}^{2}, \text { Cholesterol } \\
2.2-3 \mathrm{~g} / \mathrm{L} \text {, France }\end{array}$ & $\begin{array}{l}1.25 \mathrm{~L} \text { per day for } 8 \text { weeks } \\
\text { Bicarbonate-rich carbonated water: } \\
\mathrm{HCO}^{3-} 4168 \mathrm{mg} / \mathrm{L} \\
\mathrm{Cl}^{-} 329 \mathrm{mg} / \mathrm{L} \\
\mathrm{Na}^{+} 1626 \mathrm{mg} / \mathrm{L} \\
\mathrm{Mg}^{2+} 11 \mathrm{mg} / \mathrm{L}\end{array}$ & $\begin{array}{l}\text { Carbonated water: } \\
\mathrm{HCO}^{3-} 183 \mathrm{mg} / \mathrm{L} \\
\mathrm{Cl}^{-} 48 \mathrm{mg} / \mathrm{L} \\
\mathrm{Na}^{+} 31 \mathrm{mg} / \mathrm{L} \\
\mathrm{Mg}^{2+} 12 \mathrm{mg} / \mathrm{L}\end{array}$ & $\begin{array}{l}\text { FPG (SO) } \\
\text { Blood lipids } \\
\text { (PO) }\end{array}$ & $\begin{array}{l}\text { No sign. difference between the } \\
\text { groups for FPG. }\end{array}$ & $\begin{array}{l}\text { ITT, } 0 / 12 \\
\text { drop-outs, } \\
\text { randomization } \\
\text { and allocation not } \\
\text { reported, blinding } \\
\text { difficult due to } \\
\text { taste of water }\end{array}$ \\
\hline
\end{tabular}

PO: primary outcome; SO: secondary outcome; sign.: $p<0.05$; RCT: randomized controlled trial; FPG: fasting plasma glucose; GTT: glucose tolerance test; CVRF: cardiovascular risk factors; y: years. 
Table 5. Randomized controlled trials (RCTs) comparing low mineralized water with bicarbonate-rich water with magnesium-rich water.

\begin{tabular}{|c|c|c|c|c|c|c|c|}
\hline Author/y & Study Type & Population & Intervention & Comparison & Outcome & Results & Risk of Bias \\
\hline $\begin{array}{l}\text { Gutenbrunner, } \\
1993 \text { [16] }\end{array}$ & $\begin{array}{l}\text { RCT } \\
\text { parallel } \\
2 \text { groups } \\
\text { single-blind }\end{array}$ & $\begin{array}{l}23 \text { healthy men, } \\
19-31 \mathrm{y}, \\
\text { Germany }\end{array}$ & $\begin{array}{l}\text { 1.4 } \mathrm{L} \text { per day of the test } \\
\text { waters for } 28 \text { days } \\
\text { Bicarbonate and } \\
\text { magnesium-rich } \\
\text { carbonated water: } \\
\mathrm{HCO}^{3-} 2946 \mathrm{mg} / \mathrm{L} \\
\mathrm{Cl}^{-} 128 \mathrm{mg} / \mathrm{L} \\
\mathrm{Na}^{+} 967 \mathrm{mg} / \mathrm{L} \\
\mathrm{Mg}^{2+} 102 \mathrm{mg} / \mathrm{L}\end{array}$ & $\begin{array}{l}\text { Uncarbonated water: } \\
\mathrm{HCO}^{3-} 150 \mathrm{mg} / \mathrm{L} \\
\mathrm{Na}^{+} 19.8 \mathrm{mg} / \mathrm{L} \\
\mathrm{Mg} \text { not mentioned }\end{array}$ & $\begin{array}{l}\text { FPG } \\
\text { Fasting insulin } \\
\text { GTT } \\
\text { Fructosamin } \\
\text { (all PO) }\end{array}$ & $\begin{array}{l}\text { Bicarbonate and magnesium-rich } \\
\text { water sign. decreased glucose } \\
\text { tolerance and fructosamin compared } \\
\text { to control water, but not FPG and } \\
\text { fasting insulin. }\end{array}$ & $\begin{array}{l}\text { No ITT, } 1 / 24 \\
\text { drop-outs, single } \\
\text { blind, } \\
\text { randomization } \\
\text { and allocation not } \\
\text { reported }\end{array}$ \\
\hline $\begin{array}{l}\text { Murakami et al., } \\
2015 \text { [30] }\end{array}$ & $\begin{array}{l}\text { RCT } \\
\text { parallel } \\
2 \text { groups }\end{array}$ & $\begin{array}{l}19 \text { healthy }(7 \\
\text { men, } 12 \text { women), } \\
47 \text { y }(26-59 \text { y), } \\
\text { Japan }\end{array}$ & $\begin{array}{l}500 \mathrm{~mL} \text { per day premeal, } \\
\text { mineral or control water } \\
\text { for one week in two } \\
\text { cycles. The intervention } \\
\text { lasted } 4 \text { weeks } \\
\text { Bicarbonate and } \\
\text { magnesium-rich } \\
\text { uncarbonated water: } \\
\mathrm{HCO}^{3-} 2485 \mathrm{mg} / \mathrm{L} \\
\mathrm{Cl}^{-} 182 \mathrm{mg} / \mathrm{L} \\
\mathrm{Na}^{+} 412 \mathrm{mg} / \mathrm{L} \\
\mathrm{Mg}^{2+} 291 \mathrm{mg} / \mathrm{L}\end{array}$ & $\begin{array}{l}\text { Uncarbonated water: } \\
\mathrm{HCO}^{3-} 28 \mathrm{mg} / \mathrm{L} \\
\mathrm{Cl}^{-} 11 \mathrm{mg} / \mathrm{L} \\
\mathrm{Na}^{+} 10 \mathrm{mg} / \mathrm{L} \\
\mathrm{Mg}^{2+} 1.9 \mathrm{mg} / \mathrm{L}\end{array}$ & $\begin{array}{l}\text { FPG } \\
\text { Glycoalbumin, } \\
\text { Fasting insulin } \\
\text { HOMA-IR } \\
\text { (all PO) } \\
\text { Microbiome }\end{array}$ & $\begin{array}{l}\text { Bicarbonate and magnesium-rich } \\
\text { water sign. decreased glycoalbumin } \\
\text { compared to control water. } \\
\text { No sign. differences for FPG and } \\
\text { fasting insulin. } \\
\text { Lean-inducible bacteria was } \\
\text { increased }\end{array}$ & $\begin{array}{l}\text { No ITT, } 7 / 26 \\
\text { drop-outs, no } \\
\text { blinding }\end{array}$ \\
\hline
\end{tabular}

cardiovascular risk factors; $y$ : years. 


\section{Discussion}

\subsection{Results of RCTs Comparing Water with Other Drinks or No Drink (Table 3)}

In an RCT, 318 obese participants were advised to replace two caloric beverages (each 350-500 mL) a day with water or diet beverages for six months or to stay with the usual drinks. This resulted in a significant increase of water consumption of $1 \mathrm{~L}$ after three months and $0.8 \mathrm{~L}$ after six months and a significant decrease of fasting plasma glucose (FPG) after six months in the water group compared to the control group with no change of beverages, whereas replacement with diet beverages showed no significant difference [17].

Two trials [18,19] reported significant positive effects on body weight and glycemic parameters when replacing $250 \mathrm{~mL}$ of an diet beverage with water once a day in overweight and obese women participating in a weight loss program for 24 weeks. One trial investigated otherwise healthy women, the other trial women with well controlled type 2 diabetes. In both trials, the intake of calories and carbohydrates was significantly lower in the group drinking water instead of diet beverages, which explains at least partially the positive effect on the glycemic parameters. This result is supported by an observational study in the USA comprising 18,311 participants, which showed that a higher plain water intake was accompanied by a decrease in total intake of calories, sugary beverages, total fat, saturated fats, sugar, sodium and cholesterol [32].

Conversely, another RCT [21] comparing consumption of $1 \mathrm{~L}$ of low mineralized uncarbonated water with an diet beverage (aspartame sweetened Cola) for six months showed no significant difference between the groups in FPG, Insulin or HOMA-IR; rather, a positive trend for diet cola. This is surprising because several meta-analyses of observational studies [6,33] and a survey of 75 countries [34] found a positive correlation between diet beverages and type 2 diabetes, sometimes even higher than for sucrose sweetened beverages. Furthermore, the amount of water and duration of the intervention were sufficient, as a nine-year follow-up of 3615 participants showed a significant lower risk of hyperglycaemia (20-30\%) in participants drinking more than $0.5 \mathrm{~L}$ of water daily compared with drinking less [35], and an increase of $240 \mathrm{~mL}$ in water intake revealed a significant decline in the type 2 diabetes risk score in a cross-sectional study [36]. The authors of the study discuss the small sample size as a possible factor for this finding.

In the RCT discussed above [21], water was also compared to sucrose sweetened cola and semi-skimmed milk, both of which showed no significant difference between the groups for FPG, Insulin or HOMA-IR. The lack of a significant difference between water and cola is also surprising because two meta-analyses of observational studies show a higher incidence of type 2 diabetes with consumption of sucrose sweetened beverages [37,38], whereas the lack of a significant difference between water and milk is in accordance with the literature. A meta-analysis of observational studies on total low-fat and high-fat milk intake was not associated with an increased risk for type 2 diabetes, and yogurt intake of $80 \mathrm{~g} /$ day was even nonlinearly inversely related with a 14\% lower risk [39].

A further three studies showed no significant difference in the glycemic parameters.

One study [20] compared consumption of $1 \mathrm{~L}$ tap water per day for four weeks with drinking $1 \mathrm{~L}$ blueberry juice or no drink at all. Total calorie intake was not reported, thus it is not clear how the additional calorie intake from the juice was compensated. In a recent meta-analysis of observational studies, fruit juice consumption resulted in a 5\% increase of incidence of type 2 diabetes [6]; however, a correlation between fruit juice consumption and the incidence of type 2 diabetes was not found in a European meta-analysis [37], and an observational study from Costa Rica substituting sucrose sweetened or diet beverages with fruit juice showed a trend towards better glycemic parameters and a lower incidence of metabolic syndrome than substituting it with water [40].

Another study [22] found no significant effects when comparing the effects of drinking $230 \mathrm{~mL}$ (women) or $300 \mathrm{~mL}$ (men) red alcoholic or dealcoholized wine or water once a day in the evening over four weeks [22] on CVRF, including glycemic parameters. The amount of water may have been too small for positive effects on glycemic parameters and total calorie intake was not reported. 
The third study [23] investigated the cardiovascular effects of $150 \mathrm{~mL}$ red or white wine once a day compared to water over two years, and found no significant difference for red wine for all glycemic parameters, and for white wine even a significant decrease of FPG and HOMA-IR compared to water. The diet was comparable (Mediterranean Diet recommended) and, as the authors state in their discussion, the better result for the white wine may be a chance finding. The amount of water consumed in these studies may be too low for any effects on glycemic parameters.

The results of the last two studies are in accordance with a recent meta-analysis with moderate amounts of alcohol showing benefits on glycemic parameters [41].

In a further study (published twice) [24,31], drinking three times $150 \mathrm{~mL}$ of chamomile tea per day showed a significant decrease for $\mathrm{HbA1c}$, serum insulin and insulin sensitivity compared to $150 \mathrm{~mL}$ hot water. As chamomile tea contains the same amount of water, this study does investigate the effect of the water itself, rather that of chamomile, particularly as the additional amount of hot water was probably compensated through reduction of other drinks. The antidiabetic effect of chamomile is supported by several studies in diabetic rats, as the authors state in their discussion.

\subsection{Results of RCTs Comparing Low Mineralized Water with Bicarbonate-Rich Water with Low Magnesium} (Table 4)

One study with 18 healthy postmenopausal women showed a significant decrease of a glycemic parameter, FPG, for use of $1 \mathrm{~L}$ of carbonated bicarbonate-rich water for two months compared to $1 \mathrm{~L}$ of an uncarbonated low mineralized water [25].

The other four studies found no significant difference between the use of bicarbonate-rich water compared to low mineralized water for the investigated glycemic parameters.

One of these studies, with a sample size of 18 healthy adults, found a trend $(p=0.056)$ for FPG after eight weeks of $1 \mathrm{~L}$ of carbonated bicarbonate-rich water compared to a carbonated low mineralized water [27].

The largest study with 64 healthy adults showed a significant decrease of FPG compared to baseline for carbonated bicarbonate-rich and uncarbonated low mineralized water, with no significant difference between the groups [26]. The study showed a significant increase in total fluid intake with a significant decrease of consumption of soft drinks and fruit juice and a trend toward less beer, showing that the water was replacing other drinks. Results also showed a significant lower intake of calories and carbohydrates for both water groups. This result reflects partially a regression to the mean and partially a positive effect of substituting other beverages and food with water.

One study with 16 healthy elder adults (60-72 years) on a salt restricted diet, compared two different bicarbonate-rich waters with low mineralized water, one with a higher sodium than bicarbonate content. This trial showed no difference in FPG, and the decrease in insulin in favor of the sodium rich water (decrease of insulin -0.5 vs. $-0.9 \mathrm{mU} / \mathrm{L}$ ) was too small to reach significance [28]. In so far as this result has any significance, it is supported by a meta-analysis of 19 crossover RCTs ( $n=494)$, which found higher fasting insulin concentrations with sodium reduction [42].

The other crossover RCT, in which 12 healthy men consumed $1.25 \mathrm{~L}$ water with the highest content of bicarbonate of the searched studies $(4168 \mathrm{mg} / \mathrm{L})$ for eight weeks, also found only a small non-significant difference of $3 \mathrm{mg} / \mathrm{dL}$ for FPG in favor of the bicarbonate-rich water [29].

None of the five studies had a sample size larger than 64 participants and four studies fewer than 18 (range 12-18) and therefore did not have sufficient power to detect small or even moderate effects.

\subsection{Results of RCT Comparing Low Mineralized Water with Bicarbonate-Rich Waters with High Magnesium (Table 5)}

The first study compared consumption of $1.4 \mathrm{~L}$ of a bicarbonate-rich water with a content of $102 \mathrm{mg} / \mathrm{L}$ magnesium for four weeks with an uncarbonated low mineralized water [16]. In spite of the small sample size, the trial found a small but significant difference for serum fructosamin for the bicarbonate- and magnesium-rich water compared to the low mineralized water. Furthermore, the 
glucose tolerance test showed a significantly better result in the bicarbonate-rich water group, but there was no significant difference for the short-term glycemic parameters FPG and fasting insulin.

The other study [30] investigated a bicarbonate-rich water with an even higher concentration of magnesium $(291 \mathrm{mg} / \mathrm{L})$ and also showed a significant better result in the long-term glycemic parameter glycoalbumin compared to low mineralized water and no significance for the short-term glycemic parameters FPG, fasting insulin and the calculated HOMA-IR.

As shown below, the positive results may well be due to the high magnesium content. However, it is also apparent that the positive significant results were found only in longer-term glycemic parameters, indicating that the effects from water, especially bicarbonate-rich water, may be too small to detect them in the short-term parameters FPG, fasting insulin and HOMA-IR, but large enough to result in a moderate effect in longer terms.

The two studies $[16,30]$ had a small sample size comprising 23 and 19 healthy participants, respectively. They therefore did not have sufficient power to detect small or even moderate effects. Both studies found significant positive results for long-term glycemic parameters (fructosamin and glycoalbumin).

\subsubsection{Effect of Bicarbonate as a Supplement}

All studies comparing the effect of tap water and mineral water used bicarbonate-rich mineral water, since a high dietary acid load is associated with increased insulin resistance as well as with disturbed glucose metabolism. The following section highlights some studies investigating the effect of bicarbonate on glycemic parameters.

A recent RCT [43] in diabetic patients with chronic kidney disease showed that, compared with a placebo, supplementation with bicarbonate targeting a serum concentration of $24-28 \mathrm{mmol} / \mathrm{L}$ significantly improved multiple parameters of glucose metabolism. For example, the serum glucose concentration decreased from $127 \mathrm{mg} / \mathrm{dL}$ to $110 \mathrm{mg} / \mathrm{dL}$, the proportion of glycated haemoglobin declined from $7.7 \%$ to $6.7 \%$, insulin resistance (HOMA-IR) decreased from 6.1 to 7.0 , and serum insulin concentration decreased from $13.4 \mathrm{mcIU}$ to $19.9 \mathrm{mcIU}$. Insulin resistance declined up to a bicarbonate concentration of $28 \mathrm{mmol} / \mathrm{L}$; however, it increased when $28 \mathrm{mmol} / \mathrm{L}$ of bicarbonate was exceeded. Thus, the improvement in insulin resistance was based on a correction of acidosis rather than on the serum bicarbonate concentration itself. Mild acidosis is accompanied by increased insulin resistance even in healthy subjects.

A cross-sectional study [44] investigated the relationship between serum hydrogen carbonate concentration and insulin resistance in 1496 healthy adults. Participants with lower serum hydrogen bicarbonate concentration showed both significantly increased insulin resistance and a significantly elevated serum insulin concentration. This effect was even more distinct in participants with higher BMI. Similarly, a recent study [12] revealed a relationship between an increased lactate concentration, which is an indicator for mild metabolic acidosis, and insulin resistance in 104 sedentary adults.

The relevance of serum hydrogen carbonate in prevention was reported in a prospective study in 630 women $(\varnothing=56$ years $)$ and a 10 -year follow up. Women with lower serum hydrogen carbonate concentrations had a significant higher risk of developing type 2 diabetes than women with higher serum hydrogen carbonate concentrations [10].

\subsubsection{Effect of Mineral Rich Water in an Animal Model}

It is known that rats receiving $10 \%$ fructose in their drinking water develop symptoms of metabolic syndrome and that the plasma glucose level increases considerably.

If fructose is administered in mineral water rather than tap water, the basic parameters of glucose metabolism, e.g., glucose, insulin and triglyceride concentrations, do not deteriorate at all or at least less [45]. Similarly, rats receiving fructose in tap water showed a strong increase in aldosterone level, whereas rats receiving fructose in mineral water had the same level as the control rats that did not 
receive any fructose. A review from 2016 [46] describes the relationship between elevated aldosterone level and both metabolic syndrome and type 2 diabetes.

Sirtuin 1 (SIRT1) and peroxisome proliferator-activated receptor-gamma coactivator (PGC)1- $\alpha$ ) are proteins that counteract metabolic syndrome and aberrant glucose metabolism. SIRT1 positively influences multiple steps in glucose metabolism in the liver, the pancreas, muscles, and adipose tissue. De-acetylated PGC1- $\alpha$ is the main regulator of the SIRT1 pathway $[47,48]$. The expression of both proteins was significantly higher in liver tissue of rats receiving mineral water compared to rats receiving tap water [49]. In addition, the amount of phosphorylated insulin receptor substrate (IRS), the active form of the substrate that forwards the insulin signal, was elevated in liver tissues of rats receiving mineral water compared with rats receiving tap water [50]. This indicates increased insulin sensitivity. However, both groups of rats taking in fructose had a lower level of phosphorylated IRS compared with control rats. This might be due to the fact that the serum magnesium concentration in rats receiving fructose significantly declined $(1.71 \mathrm{mmol} / \mathrm{L}$ and $1.55 \mathrm{mmol} / \mathrm{L}$, respectively) [45].

Magnesium ions are essential for the auto-phosphorylation of the insulin receptor, as two magnesium ions bind the tyrosine kinase domain. Disruption of phosphorylation is considered the main mechanism leading to insulin resistance through lack of magnesium ions [51].

In summary, these animal experiments suggest that intake of mineral water in patients with either metabolic syndrome, pre-diabetes, or type 2 diabetes might have a positive influence.

Besides bicarbonate, magnesium seems to be important. Both RCTs studying effects of mineral water rich in magnesium determined long-term blood glucose concentrations. Hence, the positive effect observed in these studies cannot be traced back exclusively to magnesium. Still, the currently available data indicate a positive influence of magnesium on the glucose metabolism and one might assume that magnesium absorbed from mineral waters also positively influences glucose metabolism.

\subsection{Clinical Trials Considering Magnesium}

Results from studies investigating mineral waters containing relevant concentrations of magnesium are consistent with the results from studies investigating magnesium supplementation or intake of dietary magnesium. The latter demonstrated that in subjects suffering from magnesium deficiency, pre-diabetes, or insulin resistance, additional magnesium intake may ameliorate their glucose metabolism. Indeed, in $51 \%$ of the subjects suffering from magnesium deficiency and pre-diabetes, magnesium supplementation of $382 \mathrm{mg}$ /day positively influenced glucose metabolism compared to $7 \%$ of the subjects in a placebo group [52]. In adults with low insulin sensitivity, magnesium supplementation of $365 \mathrm{mg} /$ day caused the serum glucose level to drop significantly and increased insulin sensitivity [53].

The most recent systematic review from 2016 including a meta-analysis of 21 RCTs in 1362 participants (684 individuals in $\mathrm{Mg}^{2+}$ group and 678 individuals in control group) compared the influence of magnesium supplementation on insulin sensitivity and glucose metabolism in diabetic individuals with the influence in non-diabetic individuals [11]. This review demonstrated significant amelioration of insulin resistance in both diabetic and non-diabetic individuals. A significant reduction of the serum glucose concentration was observed in a subset of studies including only those with supplementation for at least four months and only considering participants with magnesium deficiency.

\subsubsection{Magnesium Blood Concentration}

In 2012 [54], a case control study in 200 obese individuals revealed that the 50 diabetics had a lower serum magnesium concentration than the 150 participating non-diabetics. Forty-eight per cent of the diabetics had magnesium deficiency compared with only $15 \%$ of the non-diabetics. Furthermore, a lower magnesium concentration was associated with a higher plasma glucose level, higher insulin resistance and a higher $\mathrm{HbAc1}$ concentration.

A prospective study explored this relationship in 8555 participants with a follow-up period of 5.7 years [55]. A reduction in the serum magnesium concentration of $0.1 \mathrm{mmol}$ was associated with 
an $18 \%$ higher risk of developing diabetes $(\mathrm{HR}=1.18 ; 95 \% \mathrm{CI}=1.04-1.33)$ and a $12 \%$ higher risk for pre-diabetes $(\mathrm{HR}=1.12 ; 95 \% \mathrm{CI}=1.01-1.25)$. In participants deficient in magnesium, the diabetes risk increased by $79 \%(\mathrm{HR}=1.79 ; 95 \% \mathrm{CI}=1.16-2.77)$ and the pre-diabetes risk increased by $44 \%(\mathrm{HR}=$ $1.44 ; 95 \% \mathrm{CI}=0.91-2.27)$, however, the latter finding was not significant.

\subsubsection{Dietary Magnesium}

In 2011, a review with meta-analysis of 13 prospective studies in a total of 536,318 participants and 24,516 diabetes cases [56] showed a significantly reduced risk for type 2 diabetes: Increasing the daily magnesium intake by $100 \mathrm{mg}$ was accompanied by a decrease in diabetes risk by $14 \%$.

The most recent meta-analysis of 25 prospective studies comprising 637,922 participants revealed the following dose-effect-correlation: An increase of daily magnesium intake by $100 \mathrm{mg}$ was associated with an $8-13 \%$ lower diabetes risk [57].

Another meta-analysis including 6 cross-sectional studies in a total of 24,473 participants investigated the correlation between magnesium intake and metabolic syndrome [58]. This study revealed that daily intake of $100 \mathrm{mg}$ magnesium was associated with a risk reduction by $17 \%$.

\subsubsection{Relevance of Magnesium for Glucose Metabolism}

A review by Gommers et al. [51] summarizes the multiple functions of magnesium on a molecular level. Another narrative review by Mooren [59] sums up the molecular mechanisms and links them to data from clinical trials.

The binding of insulin to its receptor triggers a signaling cascade leading to import of glucose into the cell (muscle), to glycogen synthesis (liver) and lipid synthesis (adipose tissue). If this signaling cascade is impaired, more insulin is released into the bloodstream. When the increased amount of insulin is in the end also unable to trigger the signaling cascade sufficiently, blood sugar levels eventually rise.

Magnesium plays a direct role in the signaling cascade as a co-factor of the insulin receptor. Magnesium binds to the intracellular domain of the insulin receptor, thereby allowing or enhancing transmission of the insulin signal induced by the extracellular binding of insulin to the receptor. Hence, magnesium increases insulin sensitivity.

It is known from studies in mice, that oral magnesium supplementation increases the number of glucose transporters in the cell membrane of muscle cells; glucose can therefore be imported faster and more effectively [60].

An inflammatory environment also contributes to development of insulin resistance and is one of the main reasons for an obesity-related elevated diabetes risk. Thus, magnesium as an anti-inflammatory ion can counteract insulin resistance [61].

To prevent diabetes, it is essential to preserve the function of $\beta$-cells [62]. A prospective study comprising 228 patients with disturbed glucose tolerance showed that the diabetes risk was smallest in participants with functioning $\beta$-cells at the beginning of the study [63]. The function of the $\beta$-cells was the strongest predictor for diabetes. In diabetic and non-diabetic participants, magnesium administration in the form of $\mathrm{MgCl}_{2}$ increased the function of $\beta$-cells [64].

Insulin also influences kidney cells to increase re-absorption of magnesium. After binding of insulin to its receptor, TRPM6 is recruited to the plasma membrane; consequently, magnesium re-absorption is enhanced [65]. Therefore, insulin resistance leads to increased magnesium excretion starting a vicious cycle.

Most of the glucose ingested from food is absorbed into muscles. Deteriorated insulin resistance inhibits absorption into the muscles, whereby the reason for deterioration may either be magnesium deficiency or acidosis, in which bicarbonate might be an issue. 


\subsection{Summary}

In summary, the evidence for positive effects of water or mineral water in improving glycemic parameters in diabetic and non-diabetic persons is low and the results are heterogenous with no clear result. Positive results were mainly seen in studies where water replaced caloric or diet beverages, and where bicarbonate and magnesium-rich mineral water and long-term glycemic parameters were used.

Meta-analyses of prospective cohort studies and other observational studies, studies with animal models and interventional studies with supplements of hydrogencarbonate and magnesium suggest a probable positive effect of drinking water and especially mineral water on glycemic parameters, supporting the positive results found in some of the RCTs, especially those substituting diet beverages or caloric beverages with water and those using bicarbonate and magnesium-rich water.

\section{Limitations and Generalizability}

The main limitation of this review is the small number of RCTs and even more the small sample sizes in most of the RCTs that give the risk of overestimating the effects. The lack of blinding in most of the RCTs gives a relative high risk of bias compared to an otherwise relative low risk of bias in most of the studies.

Most of the RCTs did not measure long-term glycemic parameters and may therefore have missed positive effects as shown in the two RCTs using fructosamin and glycoalbumin [16,30].

The interventions lasted mainly four to eight weeks, only one study lasted six months and another two years. This may have been too short, as shown in the "Da Qing Diabetes Prevention Study" with 23-year follow-up, investigating diet and physical activity, which reported that the difference in the incidence of type 2 diabetes appeared only after two years [4].

Some of the interventions used relatively little amounts of water, especially when wine was the beverage being compared, possibly leading to negative results.

In this review, the generalizability is relatively high, as we only excluded severe diseases and special circumstances as extreme physical activity, extreme climate or acute or severe diseases. In addition, the population investigated in the studies covers nearly all ages; however, there are no studies with children or adolescents.

\section{Conclusions}

Water is an ideal calorie-free and sugar-free beverage, which may be suitable to reduce calorie and sugar intake, and if high mineralized water is also used to substitute those minerals that may be relevant for bicarbonate and magnesium.

Further research should focus on long-term glycemic parameters, and larger and more rigorous studies are needed to answer the question of whether drinking water or mineral water can improve glycemic parameters in diabetic and non-diabetic persons.

Regarding the high prevalence, the associated suffering and the resulting health expenditures of type 2 diabetes, it is imperative to conduct these RCTs.

Acknowledgments: The article processing charge was funded by the German Research Foundation (DFG) and the Albert Ludwigs University Freiburg in the funding program Open Access Publishing.

Author Contributions: Johannes Naumann and Diana Biehler performed the screening, the evaluation of the studies and wrote the review. Tania Lüty supported us with the preparation and drafting of the manuscript. Catharina Sadaghiani gave us methodological support and was involved in critical revision of the review.

Conflicts of Interest: Johannes Naumann received support from balneology organisations such as Deutscher Heilbäderverband and Heilbäderverband Baden-Württemberg and is a member of these organisations. Diana Biehler received support from Verband Deutscher Mineralbrunnen. None of the organisations financed this work. The authors declare no conflict of interest. 


\section{References}

1. WHO. Diabetes. Available online: http://www.who.int/mediacentre/factsheets/fs312/en/ (accessed on 3 August 2017).

2. Gesundheitssurvey Prävalenz Häufiger Erkrankungen. Available online: http://www.gbe-bund.de/gbe10/ ergebnisse.prc_tab?fid=24742\&suchstring=Diabetes\&query_id=\&sprache=D\&fund_typ=TAB\&methode= $3 \& \mathrm{t}=1 \&$ verwandte $=1 \&$ page_ret $=0 \&$ seite $=1 \& p \_1 \mathrm{fd} \_n r=75 \& p \_n e w s=\& p \_s p r a c h k z=D \& p \_u i d=$ gast \&p aid=75552896\&hlp_nr=3\&p_janein=J\#SEARCH=\%2522Diabetes\%2522 (accessed on 13 April 2017).

3. American Diabetes Association. 5. Prevention or Delay of Type 2 Diabetes. Diabetes Care 2017, 40, S44-S47. [CrossRef]

4. Li, G.; Zhang, P.; Wang, J.; An, Y.; Gong, Q.; Gregg, E.W.; Yang, W.; Zhang, B.; Shuai, Y.; Hong, J.; et al. Cardiovascular mortality, all-cause mortality, and diabetes incidence after lifestyle intervention for people with impaired glucose tolerance in the Da Qing Diabetes Prevention Study: A 23-year follow-up study. Lancet Diabetes Endocrinol. 2014, 2, 474-480. [CrossRef]

5. O'Connor, L.; Imamura, F.; Lentjes, M.A.H.; Khaw, K.-T.; Wareham, N.J.; Forouhi, N.G. Prospective associations and population impact of sweet beverage intake and type 2 diabetes, and effects of substitutions with alternative beverages. Diabetologia 2015, 58, 1474-1483. [CrossRef] [PubMed]

6. Imamura, F.; O'Connor, L.; Ye, Z.; Mursu, J.; Hayashino, Y.; Bhupathiraju, S.N.; Forouhi, N.G. Consumption of sugar sweetened beverages, artificially sweetened beverages, and fruit juice and incidence of type 2 diabetes: Systematic review, meta-analysis, and estimation of population attributable fraction. BMJ 2015, 351, h3576. [CrossRef] [PubMed]

7. Gibson, S.A.; Horgan, G.W.; Francis, L.E.; Gibson, A.A.; Stephen, A.M. Low calorie beverage consumption is associated with energy and nutrient intakes and diet quality in British adults. Nutrients 2016, 8, 9. [CrossRef] [PubMed]

8. Carroll, H.A.; Betts, J.A.; Johnson, L. An investigation into the relationship between plain water intake and glycated $\mathrm{Hb}(\mathrm{HbA} 1 \mathrm{c})$ : A sex-stratified, cross-sectional analysis of the UK National Diet and Nutrition Survey (2008-2012). Br. J. Nutr. 2016, 116, 1770-1780. [CrossRef] [PubMed]

9. Stookey, J.J.D. Negative, null and beneficial effects of drinking water on energy intake, energy expenditure, fat oxidation and weight change in randomized trials: A qualitative review. Nutrients 2016, 8, 19. [CrossRef] [PubMed]

10. Mandel, E.I.; Curhan, G.C.; Hu, F.B.; Taylor, E.N. Plasma bicarbonate and risk of type 2 diabetes mellitus. CMAJ Can. Med. Assoc. J. 2012, 184, E719-E725. [CrossRef] [PubMed]

11. Simental-Mendía, L.E.; Sahebkar, A.; Rodríguez-Morán, M.; Guerrero-Romero, F. A systematic review and meta-analysis of randomized controlled trials on the effects of magnesium supplementation on insulin sensitivity and glucose control. Pharmacol. Res. 2016, 111, 272-282. [CrossRef] [PubMed]

12. Williams, R.S.; Heilbronn, L.K.; Chen, D.L.; Coster, A.C.F.; Greenfield, J.R.; Samocha-Bonet, D. Dietary acid load, metabolic acidosis and insulin resistance-Lessons from cross-sectional and overfeeding studies in humans. Clin. Nutr. 2016, 35, 1084-1090. [CrossRef] [PubMed]

13. Souto, G.; Donapetry, C.; Calviño, J.; Adeva, M.M. Metabolic acidosis-induced insulin resistance and cardiovascular risk. Metab. Syndr. Relat. Disord. 2011, 9, 247-253. [CrossRef] [PubMed]

14. Rydén, L.; Grant, P.J.; Anker, S.D.; Berne, C.; Cosentino, F.; Danchin, N.; Deaton, C.; Escaned, J.; Hammes, H.P.; Huikuri, H.; et al. ESC Guidelines on diabetes, pre-diabetes, and cardiovascular diseases developed in collaboration with the EASD—Summary. Diabetes Vasc. Dis. Res. 2014, 11, 133-173. [CrossRef]

15. Higgins, J.P.T.; Altman, D.G.; Gøtzsche, P.C.; Jüni, P.; Moher, D.; Oxman, A.D.; Savovic, J.; Schulz, K.F.; Weeks, L.; Sterne, J.A.C.; et al. The Cochrane Collaboration's tool for assessing risk of bias in randomised trials. BMJ 2011, 343, d5928. [CrossRef] [PubMed]

16. Gutenbrunner, C. Kontrollierte Studie über die Wirkung einer Haustrinkkur mit einem Natrium-Hydrogencarbonat-Säuerling auf die Blutzuckerregulation bei gesunden Versuchspersonen. Phys. Med. Rehabil. Kurortmed. 1993, 3, 108-110. [CrossRef]

17. Tate, D.F.; Turner-McGrievy, G.; Lyons, E.; Stevens, J.; Erickson, K.; Polzien, K.; Diamond, M.; Wang, X.; Popkin, B. Replacing caloric beverages with water or diet beverages for weight loss in adults: Main results of the Choose Healthy Options Consciously Everyday (CHOICE) randomized clinical trial. Am. J. Clin. Nutr. 2012, 95, 555-563. [CrossRef] [PubMed] 
18. Madjd, A.; Taylor, M.A.; Delavari, A.; Malekzadeh, R.; Macdonald, I.A.; Farshchi, H.R. Effects on weight loss in adults of replacing diet beverages with water during a hypoenergetic diet: A randomized, 24-wk clinical trial. Am. J. Clin. Nutr. 2015, 102, 1305-1312. [CrossRef] [PubMed]

19. Madjd, A.; Taylor, M.A.; Delavari, A.; Malekzadeh, R.; Macdonald, I.A.; Farshchi, H.R. Beneficial effects of replacing diet beverages with water on type 2 diabetic obese women following a hypo-energetic diet: A randomized, 24-week clinical trial. Diabetes Obes. Metab. 2017, 19, 125-132. [CrossRef] [PubMed]

20. Tonstad, S.; Klemsdal, T.O.; Landaas, S.; Høieggen, A. No effect of increased water intake on blood viscosity and cardiovascular risk factors. Br. J. Nutr. 2006, 96, 993-996. [CrossRef] [PubMed]

21. Maersk, M.; Belza, A.; Stødkilde-Jørgensen, H.; Ringgaard, S.; Chabanova, E.; Thomsen, H.; Pedersen, S.B.; Astrup, A.; Richelsen, B. Sucrose-sweetened beverages increase fat storage in the liver, muscle, and visceral fat depot: A 6-mo randomized intervention study. Am. J. Clin. Nutr. 2012, 95, 283-289. [CrossRef] [PubMed]

22. Mori, T.A.; Burke, V.; Zilkens, R.R.; Hodgson, J.M.; Beilin, L.J.; Puddey, I.B. The effects of alcohol on ambulatory blood pressure and other cardiovascular risk factors in type 2 diabetes: A randomized intervention. J. Hypertens. 2016, 34, 421-428; discussion 428. [CrossRef] [PubMed]

23. Gepner, Y.; Golan, R.; Harman-Boehm, I.; Henkin, Y.; Schwarzfuchs, D.; Shelef, I.; Durst, R.; Kovsan, J.; Bolotin, A.; Leitersdorf, E.; et al. Effects of initiating moderate alcohol intake on cardiometabolic risk in adults with type 2 diabetes: A 2-year randomized, controlled trial. Ann. Intern. Med. 2015, 163, 569-579. [CrossRef] [PubMed]

24. Rafraf, M.; Zemestani, M.; Asghari-Jafarabadi, M. Effectiveness of chamomile tea on glycemic control and serum lipid profile in patients with type 2 diabetes. J. Endocrinol. Invesig. 2015, 38, 163-170. [CrossRef] [PubMed]

25. Schoppen, S.; Pérez-Granados, A.M.; Carbajal, Á.; Oubiña, P.; Sánchez-Muniz, F.J.; Gómez-Gerique, J.A.; Vaquero, M.P. A sodium-rich carbonated mineral water reduces cardiovascular risk in postmenopausal women. J. Nutr. 2004, 134, 1058-1063. [PubMed]

26. Toxqui, L.; Vaquero, M.P. An intervention with mineral water decreases cardiometabolic risk biomarkers. A crossover, randomised, controlled trial with two mineral waters in moderately hypercholesterolaemic adults. Nutrients 2016, 8, 400. [CrossRef] [PubMed]

27. Pérez-Granados, A.M.; Navas-Carretero, S.; Schoppen, S.; Vaquero, M.P. Reduction in cardiovascular risk by sodium-bicarbonated mineral water in moderately hypercholesterolemic young adults. J. Nutr. Biochem. 2010, 21, 948-953. [CrossRef] [PubMed]

28. Schorr, U.; Distler, A.; Sharma, A.M. Effect of sodium chloride- and sodium bicarbonate-rich mineral water on blood pressure and metabolic parameters in elderly normotensive individuals: A randomized double-blind crossover trial. J. Hypertens. 1996, 14, 131-135. [PubMed]

29. Zair, Y.; Kasbi-Chadli, F.; Housez, B.; Pichelin, M.; Cazaubiel, M.; Raoux, F.; Ouguerram, K. Effect of a high bicarbonate mineral water on fasting and postprandial lipemia in moderately hypercholesterolemic subjects: A pilot study. Lipids Health Dis. 2013, 12, 105. [CrossRef] [PubMed]

30. Murakami, S.; Goto, Y.; Ito, K.; Hayasaka, S.; Kurihara, S.; Soga, T.; Tomita, M.; Fukuda, S. The consumption of bicarbonate-rich mineral water improves glycemic control. Evid.-Based Complement. Altern. Med. 2015, 2015, 824395. [CrossRef] [PubMed]

31. Zemestani, M.; Rafraf, M.; Asghari-Jafarabadi, M. Chamomile tea improves glycemic indices and antioxidants status in patients with type 2 diabetes mellitus. Nutrition 2016, 32, 66-72. [CrossRef] [PubMed]

32. An, R.; McCaffrey, J. Plain water consumption in relation to energy intake and diet quality among US adults, 2005-2012. J. Hum. Nutr. Diet. 2016, 29, 624-632. [CrossRef] [PubMed]

33. Greenwood, D.C.; Threapleton, D.E.; Evans, C.E.L.; Cleghorn, C.L.; Nykjaer, C.; Woodhead, C.; Burley, V.J. Association between sugar-sweetened and artificially sweetened soft drinks and type 2 diabetes: Systematic review and dose-response meta-analysis of prospective studies. Br. J. Nutr. 2014, 112, 725-734. [CrossRef] [PubMed]

34. Basu, S.; McKee, M.; Galea, G.; Stuckler, D. Relationship of soft drink consumption to global overweight, obesity, and diabetes: A cross-national analysis of 75 countries. Am. J. Public Health 2013, 103, 2071-2077. [CrossRef] [PubMed]

35. Roussel, R.; Fezeu, L.; Bouby, N.; Balkau, B.; Lantieri, O.; Alhenc-Gelas, F.; Marre, M.; Bankir, L.; DESIR Study Group. Low water intake and risk for new-onset hyperglycemia. Diabetes Care 2011, 34, 2551-2554. [CrossRef] [PubMed] 
36. Carroll, H.A.; Davis, M.G.; Papadaki, A. Higher plain water intake is associated with lower type 2 diabetes risk: A cross-sectional study in humans. Nutr. Res. 2015, 35, 865-872. [CrossRef] [PubMed]

37. Consortium, T.I. Consumption of sweet beverages and type 2 diabetes incidence in European adults: Results from EPIC-InterAct. Diabetologia 2013, 56, 1520-1530. [CrossRef] [PubMed]

38. Malik, V.S.; Popkin, B.M.; Bray, G.A.; Després, J.-P.; Willett, W.C.; Hu, F.B. Sugar-sweetened beverages and risk of metabolic syndrome and type 2 diabetes: A meta-analysis. Diabetes Care 2010, 33, 2477-2483. [CrossRef] [PubMed]

39. Gijsbers, L.; Ding, E.L.; Malik, V.S.; Goede, J.D.; Geleijnse, J.M.; Soedamah-Muthu, S.S. Consumption of dairy foods and diabetes incidence: A dose-response meta-analysis of observational studies. Am. J. Clin. Nutr. 2016, 103, 1111-1124. [CrossRef] [PubMed]

40. Mattei, J.; Malik, V.; Hu, F.B.; Campos, H. Substituting homemade fruit juice for sugar-sweetened beverages is associated with lower odds of metabolic syndrome among Hispanic adults. J. Nutr. 2012, 142, 1081-1087. [CrossRef] [PubMed]

41. Baliunas, D.O.; Taylor, B.J.; Irving, H.; Roerecke, M.; Patra, J.; Mohapatra, S.; Rehm, J. Alcohol as a risk factor for type 2 diabetes: A systematic review and meta-analysis. Diabetes Care 2009, 32, 2123-2132. [CrossRef] [PubMed]

42. Patel, S.M.; Cobb, P.; Saydah, S.; Zhang, X.; de Jesus, J.M.; Cogswell, M.E. Dietary sodium reduction does not affect circulating glucose concentrations in fasting children or adults: Findings from a systematic review and meta-analysis. J. Nutr. 2015, 145, 505-513. [CrossRef] [PubMed]

43. Bellasi, A.; Di Micco, L.; Santoro, D.; Marzocco, S.; De Simone, E.; Cozzolino, M.; Di Lullo, L.; Guastaferro, P.; Di Iorio, B.; UBI Study Investigators. Correction of metabolic acidosis improves insulin resistance in chronic kidney disease. BMC Nephrol. 2016, 17, 158. [CrossRef] [PubMed]

44. Farwell, W.R.; Taylor, E.N. Serum bicarbonate, anion gap and insulin resistance in the National Health and Nutrition Examination Survey. Diabet. Med. 2008, 25, 798-804. [CrossRef] [PubMed]

45. Pereira, C.D.; Severo, M.; Araújo, J.R.; Guimarães, J.T.; Pestana, D.; Santos, A.; Ferreira, R.; Ascensão, A.; Magalhães, J.; Azevedo, I.; et al. Relevance of a hypersaline sodium-rich naturally sparkling mineral water to the protection against metabolic syndrome induction in fructose-fed sprague-dawley rats: A biochemical, metabolic, and redox approach. Int. J. Endocrinol. 2014, 2014, 384583. [CrossRef] [PubMed]

46. Das, U.N. Renin-angiotensin-aldosterone system in insulin resistance and metabolic syndrome. J. Transl. Intern. Med. 2016, 4, 66-72. [CrossRef] [PubMed]

47. Guclu, A.; Erdur, F.M.; Turkmen, K. The emerging role of sirtuin 1 in cellular metabolism, diabetes mellitus, diabetic kidney disease and hypertension. Exp. Clin. Endocrinol. Diabetes 2016, 124, 131-139. [CrossRef] [PubMed]

48. Rodgers, J.T.; Lerin, C.; Haas, W.; Gygi, S.P.; Spiegelman, B.M.; Puigserver, P. Nutrient control of glucose homeostasis through a complex of PGC-1 $\alpha$ and SIRT1. Nature 2005, 434, 113-118. [CrossRef] [PubMed]

49. Pereira, C.D.; Severo, M.; Neves, D.; Ascensão, A.; Magalhães, J.; Guimarães, J.T.; Monteiro, R.; Martins, M.J. Natural mineral-rich water ingestion improves hepatic and fat glucocorticoid-signaling and increases sirtuin $1 \mathrm{in}$ an animal model of metabolic syndrome. Horm. Mol. Biol. Clin. Investig. 2015, 21, 149-157. [CrossRef] [PubMed]

50. Pereira, C.D.; Passos, E.; Severo, M.; Vitó, I.; Wen, X.; Carneiro, F.; Gomes, P.; Monteiro, R.; Martins, M.J. Ingestion of a natural mineral-rich water in an animal model of metabolic syndrome: Effects in insulin signaling and endoplasmic reticulum stress. Horm. Mol. Biol. Clin. Investig. 2016, 26, 135-150. [CrossRef] [PubMed]

51. Gommers, L.M.M.; Hoenderop, J.G.J.; Bindels, R.J.M.; de Baaij, J.H.F. Hypomagnesemia in type 2 diabetes: A vicious circle? Diabetes 2016, 65, 3-13. [CrossRef] [PubMed]

52. Guerrero-Romero, F.; Simental-Mendía, L.E.; Hernández-Ronquillo, G.; Rodriguez-Morán, M. Oral magnesium supplementation improves glycaemic status in subjects with prediabetes and hypomagnesaemia: A double-blind placebo-controlled randomized trial. Diabetes Metab. 2015, 41, 202-207. [CrossRef] [PubMed]

53. Mooren, F.C.; Krüger, K.; Völker, K.; Golf, S.W.; Wadepuhl, M.; Kraus, A. Oral magnesium supplementation reduces insulin resistance in non-diabetic subjects-A double-blind, placebo-controlled, randomized trial. Diabetes Obes. Metab. 2011, 13, 281-284. [CrossRef] [PubMed]

54. Lecube, A.; Baena-Fustegueras, J.A.; Fort, J.M.; Pelegri, D.; Hernandez, C.; Simo, R. Diabetes is the main factor accounting for hypomagnesemia in obese subjects. PLoS ONE 2012, 7, e30599. [CrossRef] [PubMed] 
55. Kieboom, B.C.T.; Ligthart, S.; Dehghan, A.; Kurstjens, S.; de Baaij, J.H.F.; Franco, O.H.; Hofman, A.; Zietse, R.; Stricker, B.H.; Hoorn, E.J. Serum magnesium and the risk of prediabetes: A population-based cohort study. Diabetologia 2017, 60, 843-853. [CrossRef] [PubMed]

56. Dong, J.-Y.; Xun, P.; He, K.; Qin, L.-Q. Magnesium intake and risk of type 2 diabetes: Meta-analysis of prospective cohort studies. Diabetes Care 2011, 34, 2116-2122. [CrossRef] [PubMed]

57. Fang, X.; Han, H.; Li, M.; Liang, C.; Fan, Z.; Aaseth, J.; He, J.; Montgomery, S.; Cao, Y. Dose-response relationship between dietary magnesium intake and risk of type 2 diabetes mellitus: A systematic review and meta-regression analysis of prospective cohort studies. Nutrients 2016, 8, 739. [CrossRef] [PubMed]

58. Dibaba, D.T.; Xun, P.; Fly, A.D.; Yokota, K.; He, K. Dietary magnesium intake and risk of metabolic syndrome: A meta-analysis. Diabet. Med. 2014, 31, 1301-1309. [CrossRef] [PubMed]

59. Mooren, F.C. Magnesium and disturbances in carbohydrate metabolism. Diabetes Obes. Metab. 2015, 17, 813-823. [CrossRef] [PubMed]

60. Ha, B.G.; Park, J.-E.; Shin, E.J.; Shon, Y.H. Modulation of glucose metabolism by balanced deep-sea water ameliorates hyperglycemia and pancreatic function in streptozotocin-induced diabetic mice. PLoS ONE 2014, 9, e102095. [CrossRef] [PubMed]

61. Dibaba, D.T.; Xun, P.; He, K. Dietary magnesium intake is inversely associated with serum C-reactive protein levels: Meta-analysis and systematic review. Eur. J. Clin. Nutr. 2014, 68, 510-516. [CrossRef] [PubMed]

62. Buchanan, T.A.; Xiang, A.H.; Peters, R.K.; Kjos, S.L.; Marroquin, A.; Goico, J.; Ochoa, C.; Tan, S.; Berkowitz, K.; Hodis, H.N.; et al. Preservation of pancreatic beta-cell function and prevention of type 2 diabetes by pharmacological treatment of insulin resistance in high-risk hispanic women. Diabetes 2002, 51, $2796-2803$. [CrossRef] [PubMed]

63. Defronzo, R.A.; Tripathy, D.; Schwenke, D.C.; Banerji, M.; Bray, G.A.; Buchanan, T.A.; Clement, S.C.; Henry, R.R.; Kitabchi, A.E.; Mudaliar, S.; et al. Prediction of diabetes based on baseline metabolic characteristics in individuals at high risk. Diabetes Care 2013, 36, 3607-3612. [CrossRef] [PubMed]

64. Guerrero-Romero, F.; Rodríguez-Morán, M. Magnesium improves the beta-cell function to compensate variation of insulin sensitivity: Double-blind, randomized clinical trial. Eur. J. Clin. Investig. 2011, 41, 405-410. [CrossRef] [PubMed]

65. Nair, A.V.; Hocher, B.; Verkaart, S.; van Zeeland, F.; Pfab, T.; Slowinski, T.; Chen, Y.-P.; Schlingmann, K.P.; Schaller, A.; Gallati, S.; et al. Loss of insulin-induced activation of TRPM6 magnesium channels results in impaired glucose tolerance during pregnancy. Proc. Natl. Acad. Sci. USA 2012, 109, 11324-11329. [CrossRef] [PubMed]

(C) 2017 by the authors. Licensee MDPI, Basel, Switzerland. This article is an open access article distributed under the terms and conditions of the Creative Commons Attribution (CC BY) license (http://creativecommons.org/licenses/by/4.0/). 